\title{
NKG2D-IL-15 fusion protein encapsulated in N-[(2-hydroxy-3- trimethylammonium) propyl] chitosan chloride retards melanoma growth in mice
}

\author{
Rong Chen ${ }^{1 \#}$, Yimei Ding ${ }^{1 \#}$, Juqun Xi ${ }^{1}$, Guotao Lu $^{2}$, Weiming Xiao ${ }^{2,3}$, Yanbing Ding ${ }^{2}$, Li Qian ${ }^{1,4}$, Zhijie Lin ${ }^{1,3,4,5}$, \\ Weijuan Gong ${ }^{1,2,3,4,5}$
}

${ }^{1}$ Department of Immunology, Institute of Translational Medicine, Medical College, ${ }^{2}$ Department of Gastroenterology, Affiliated Hospital, ${ }^{3}$ Jiangsu Key Laboratory of Integrated Traditional Chinese and Western Medicine for Prevention and Treatment of Senile Diseases, ${ }^{4}$ Jiangsu Co-innovation Center for Prevention and Control of Important Animal Infectious Diseases and Zoonoses, Jjiangsu Key Laboratory of Zoonosis, Yangzhou University, Yangzhou 225009, China

Contributions: (I) Conception and design: W Gong; (II) Administrative support: W Xiao, Y Ding, L Qian; (III) Provision of study materials or patients: J Xi, G Lu; (IV) Collection and assembly of data: Z Lin, R Chen, Y Ding; (V) Data analysis and interpretation: Z Lin; (VI) Manuscript writing: All authors; (VII) Final approval of manuscript: All authors.

\#These authors contributed equally to this work.

Correspondence to: Weijuan Gong; Zhijie Lin. Department of Immunology, Institute of Translational Medicine, Medical College, Yangzhou University, Yangzhou 225009, China. Email: wjgong@yzu.edu.cn; zjielin@yzu.edu.cn.

Background: Chitosan can be modified to increase the efficiency of the delivery of chemical drugs, nucleic acids, or proteins. Sodium tripolyphosphate (TPP) is a noncytotoxic and polyanionic crosslinker that binds with the positively charged ions of chitosan. DsNKG2D-IL-15 is a fusion protein that exerts promising antitumor effects via lymphocyte activation. The extracellular domains of double NKG2D is linked to IL-15. Methods: To increase the stability and efficiency of dsNKG2D-IL-15 protein, the fusion protein was encapsulated in nanoparticles based on chitosan pre-modified with $\mathrm{N}$-(2-hydroxy) propyl-3-trimethyl ammonium (HTCC). Moreover, the biological activity of protein nanoparticle was evaluated on the mouse lymphocyte ex vivo and mouse tumor model in vivo.

Results: TPP sharply promoted the HTCC chitosan encapsulating efficiency (85-95\%) with dsNKG2DIL-15. The protein nanoparticle displayed a spherical shape with a diameter of 200-400 nm and zetapotential value of $15.6 \pm 4.82 \mathrm{mV}$. DsNKG2D-IL-15 could be released from the nanogel within $72 \mathrm{~h}$. In addition, the protein biological activity for lymphocyte activation was maintained. Natural killer (NK) and $\mathrm{CD}^{+} \mathrm{T}$ cells increased the activity of IFN- $\gamma$ production and degranulation after incubation with the dsNKG2D-IL-15-HTCC-TPP nanoparticle ex vivo. Treatment with dsNKG2D-IL-15 nanoparticles exhibited better effects of inhibiting tumor growth and prolonging the life span of B16BL6-MICA tumorbearing mice in vivo than by using the dsNKG2D-IL-15 protein alone.

Conclusions: The dsNKG2D-IL-15 protein nanoparticle exhibited notable effects of lymphocyte activation and tumor inhibition. The protein nanoparticle could be developed further for tumor therapy in clinical practice.

Keywords: Nanoparticle; chitosan; NKG2D; IL-15; melanoma

Submitted Apr 02, 2019. Accepted for publication Aug 27, 2019.

doi: $10.21037 /$ tcr.2019.09.36

View this article at: http://dx.doi.org/10.21037/tcr.2019.09.36 


\section{Introduction}

Chitosan has good biological activity and biocompatibility and can be absorbed by the body. Chitosan has been widely used as a carrier for the delivery of biological substances due to its natural features of linear and cationic polymer of glucosamine and $\mathrm{N}$-acetyl-glucosamine. However, chitosan is only soluble in acidic medium because of its $\mathrm{pKa}$ value of approximately 6.5. The amino groups of chitosan are only partially protonated in neutral and physiological environments, thereby leading to nonsufficient binding with most physiological proteins. N-(2-hydroxy) propyl3 -trimethyl ammonium chitosan chloride (HTCC) is a quaternary chitosan that has been constructed to increase the positive charge and solubility $(1,2)$.

The recombinant fusion protein of NKG2D-IL-15 had been previously generated in our laboratory $(3,4)$. NKG2D binds to major histocompatibility complex (MHC) I chainassociated antigens A and B (MICA/MICB) or UL-16 binding proteins (ULBP) in humans. The NKG2D ligands of mouse consist of retinoic acid early inducible- 1 gene (RAE-1), H60, and murine UL16-binding protein-like transcript (MULT)-1. The NKG2D ligands are generally expressed by tumor or stress-associated cells $(5,6)$. IL-15 is an important cytokine for activation, proliferation, and survival of natural killer $(\mathrm{NK})$ and $\mathrm{CD} 8^{+} \mathrm{T}$ cells $(7,8)$. The double gene fragments of extracellular domains of NKG2D were connected with the interleukin (IL)-15 gene and inserted into a prokaryotic expression vector. The fusion protein, called dsNKG2D-IL-15, was expressed at a large quantity and isolated for bioactivity evaluation. Both human and mouse dsNKG2D-IL-15 displayed higher activity against tumor growth than IL-15. DsNKG2D-IL-15 not only was efficiently distributed in tumor tissues but also able to promote several $\mathrm{NK}$ and $\mathrm{CD} 8^{+} \mathrm{T}$ cells to inhibit tumor growth $(3,4)$.

We also constructed the recombinant eukaryotic expression vectors of pcDNA3.1(-)-dsNKG2D-IL-15 (9) and pcDNA3.1(-)-dsNKG2D-IL-21 (10). These DNA sequences were delivered by nanoparticles based on chitosan. Two nanoparticle gene vaccines showed favorable effects on tumor growth and prolonged survival of tumor-bearing mice. The dsNKG2D-IL-2 1 gene nanoparticle-vaccine had been shown to have a tumortargeted distribution due to the enhanced permeability and retention (EPR) effect of nanoparticle in tumors (10). However, when the gene nanoparticle vaccine is used, the nanoparticle must be taken up first, and the phagocytosed gene should efficiently encode the corresponding protein in the cytoplasm. Here, we generated an HTCC-sodium tripolyphosphate (TPP) nanoparticle for delivery of the dsNKG2D-IL-15 fusion protein. The physical, chemical, and release features and also the antitumor activity in vivo of this protein nanoparticle were also evaluated.

\section{Methods}

\section{Reagents, antibodies, and cell lines}

The human dsNKG2D-IL-15 fusion protein was generated as described before (3) and adjusted to a concentration of $1 \mathrm{mg} / \mathrm{mL}$. Chitosan (MW $20 \mathrm{kDa}$ ) with around 85\% deacetylation was purchased from Yuhuan County Marine Chemical Company (Yuhuan, Zhejiang, China). TPP was from Sinopharm (Shanghai, China). Antibodies against CD8 (53.67), NK1.1 (PK136), CD69 (LG.3A10), NKG2D (CX5), CD107a (1D4B), and CD44 (IM7) were all purchased from Biolegend (San Diego, CA, USA). YAC-1, RAW264.7 cell lines were obtained from ATCC. B16 cells with stable ectopic expression of MICA were constructed and stored regularly.

\section{Generation of HTCC}

HTCC was prepared according to previous method $(11,12)$. Briefly, $2 \mathrm{~g}$ of chitosan powder was suspended in deionized water containing $2 \%$ HAc, and then the mixture was stirred for 30 min prior to dropwise addition of glycidyltrimethylammonium chloride (GTMAC) with continuous stirring. The reaction mixture was then stirred at $80{ }^{\circ} \mathrm{C}$ for $6 \mathrm{~h}$, and the product was collected by filtration after being precipitated and washed by hot alcohol. The obtained product was dissolved in distilled water, dialyzed against distilled water for 5 days, and lyophilized. The final polymer was HTCC.

\section{Preparation of HTCC-dsNKG2D-IL-15 nanoparticles}

TPP was first added into the HTCC solution $(1 \mathrm{mg} / \mathrm{mL})$ at a mass ratio of 1:10 (13). The recombinant dsNKG2DIL-15 protein was put into the solution at a mass ratio (chitosan:protein) of 1:1, 1:2, or 2:1, and the mixed solution was completely shaken at room temperature for $1 \mathrm{~h}$. Afterward, the solution was centrifuged for $30 \mathrm{~min}$ at the speed of $12,000 \mathrm{~g}$. The precipitate and supernatant were separated. The supernatant was collected for the detection of the content of free protein by a spectrophotometer. The 
free protein was also confirmed by sodium dodecyl sulfate polyacrylamide gel electrophoresis (SDS-PAGE). The pellet was re-suspended with sterile PBS buffer for future use. The encapsulation efficiency was calculated by the following formula: encapsulation rate $=$ (total amount of recombinant protein - free protein content)/total amount of recombinant protein $\times 100 \%$.

\section{Characterization of HTCC-dsNKG2D-IL-15 nanoparticles}

The sizes of the nanoparticles were measured by an electron microscope (Tecnai 12, Phillip, The Netherlands). Aqueous solutions of HTCC/protein nanoparticles were analyzed by dynamic light scattering and zeta potential measurements (Zetasizer Nano ZS 90, Malvern, UK) to determine their particle size and zeta potential.

\section{Protein release from nanoparticles}

The HTCC nanoparticles loaded with dsNKG2D-IL-15 protein were put into a small dialysis bag with $220 \mu \mathrm{m}$ filter and then placed in a sterile phosphate buffer saline (PBS). After gentle stirring, buffer outside the dialysis bag was collected at 1, 2, 4, 8, 24, 48, and $72 \mathrm{~h}$. After being centrifuged with tubes ( $30 \mathrm{kD}$ aperture), the dialyzed buffers were concentrated and analyzed by SDS-PAGE.

\section{Cell cytotoxicity}

RAW264.7 and B16BL6 cells in the logarithmic phase were divided into seven groups, which consisted of culture medium, vacant HTCC $(1.25,2.5$, and $5 \mu \mathrm{g})$, and HTCC protein $(\mathrm{HTCC}=1.25,2.5$, and $5 \mu \mathrm{g}$, chitosan: protein $=1: 1$ ). Nanoparticles were incubated with RAW264.7 or B16BL6 cells for $5 \mathrm{~d}$. Then, the MTS reagent from Promega (Madison, WI, USA) was mixed with PMS solution with a volume ratio of $20: 1$ and added into cell culture system. The color change was detected within 1-4 h, and $\mathrm{OD}_{490}$ value was determined by a microplate reader.

\section{Activation of $\mathrm{NK}$ and $\mathrm{CD} 8^{+} \mathrm{T}$ cells by nanoparticles}

Mouse mononuclear cells $\left(1 \times 10^{6}\right)$ of spleen were incubated with vacant $\mathrm{HTCC}(1.25,2.5$, and $5 \mu \mathrm{g})$ or HTCC nanoparticles encapsulated with dsNKG2D-IL-15 protein $(\mathrm{HTCC}=1.25,2.5$, and $5 \mu \mathrm{g}$, chitosan: protein $=1: 1)$ for
$24 \mathrm{~h}$. The recombinant dsNKG2D-IL-15 protein was also used to stimulate splenocytes as a positive control. Proliferation of splenocytes was determined by the cell numbers of culture systems and assayed by the MTS/PMS kit described previously. The IFN- $\gamma$ production of NK $\left(\mathrm{CD} 3^{-} \mathrm{NK} 1.1^{+}\right)$or $\mathrm{CD}^{+}{ }^{+} \mathrm{T}\left(\mathrm{CD}^{+}{ }^{+} \mathrm{CD} 8^{+}\right)$cells was detected by flow-cytometry intracellular staining with its monoclonal antibody. For the analysis of degranulation activity of NK or $\mathrm{CD}^{+} \mathrm{T}$ cells, YAC-1 or B16BL6-MICA cells as target cells were mixed with $\mathrm{NK}$ or $\mathrm{CD}^{+} \mathrm{T}$ cells at a ratio of 1:3. After $2 \mathrm{~h}$, monensin (10 $\mu \mathrm{m}$, GolgiStop; BD Biosciences, San Jose, CA, USA) was added for another $2 \mathrm{~h}$. Then, cells were collected to beco-stained with NK1.1 or CD8 antibody and CD107a antibody and analyzed by flow cytometry.

\section{Mouse tumor models}

B16BL6-MICA cells $\left(2 \times 10^{6}\right)$ in log phase were subcutaneously injected into the back of C57BL/6 mice. After 5 days of inoculation, mice were injected intraperitoneally with PBS, vacant HTCC $(50 \mu \mathrm{g})$, HTCC-dsNKG2D-IL-15 nanoparticle (HTCC $=50 \mu \mathrm{g}$, chitosan :protein $=1: 1$ ), or dsNKG2D-IL-15 protein $(50 \mu \mathrm{g})$ every 3 days. The volume of tumor was measured according to the following formula: $\mathrm{V}=1 / 2 \mathrm{ab}^{2}$ (The longest diameter of the mouse subcutaneous tumor is a, and the vertical diameter is b) every 2 days. The survival of each mouse was also observed and recorded. Some tumor-bearing mice were sacrificed to analyze the phenotypic changes of spleen $\mathrm{NK}\left(\mathrm{CD} 3^{-} \mathrm{NK} 1.1^{+}\right)$or $\mathrm{CD} 8^{+}$ $\mathrm{T}\left(\mathrm{CD}^{+}{ }^{+} \mathrm{CD} 8^{+}\right)$cells after different treatments.

\section{Statistical analysis}

ANOVA was used to compare the differences among more than two groups. The differences of two groups were analyzed by group Student's $t$-test. Survival curves were plotted by Kaplan-Meier analysis. All analyses were carried out with the GraphPad Prism software. $\mathrm{P}<0.05$ was considered statistically significant.

\section{Results}

\section{Characterization of HTCC-based nanoparticles}

The HTCC molecular structure is shown in Figure 1A. After HTCC was synthesized from the original chitosan, we used HTCC to encapsulate dsNKG2D-IL-15 protein at the serial different ratios. Free proteins of supernatants 
A
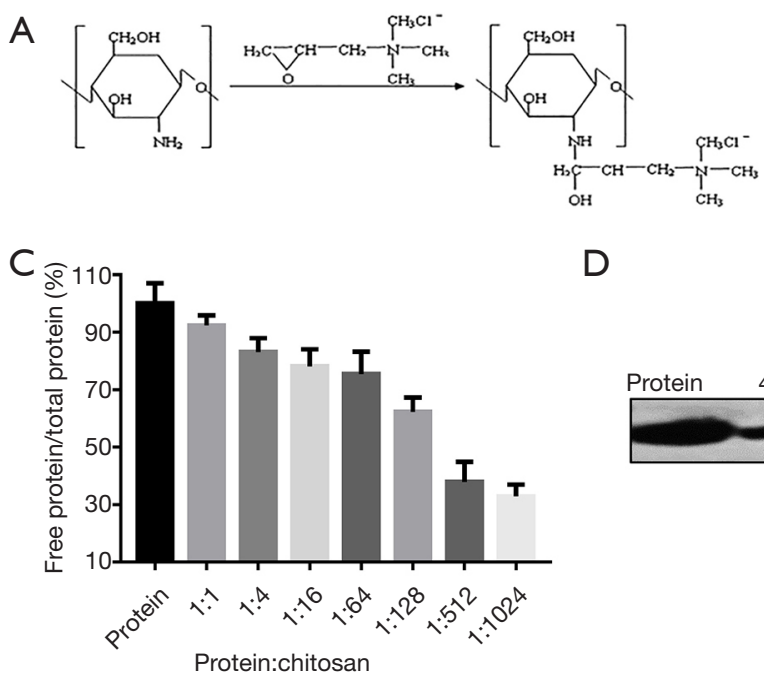

B

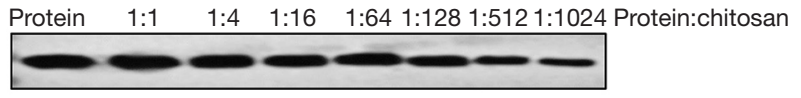

$\mathrm{D}$

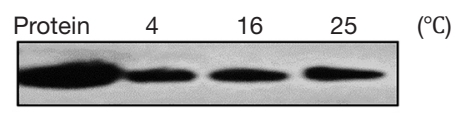

E

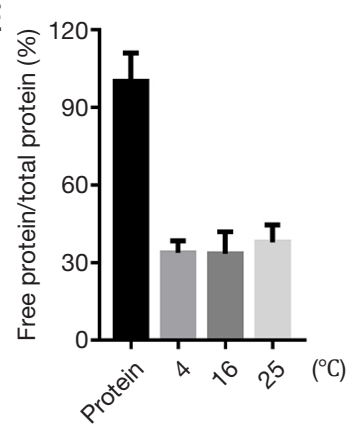

Figure 1 Preparation of protein chitosan nanoparticle based on HTCC. (A) Molecular structure of HTCC; (B) SDS-PAGE analysis of free proteins in supernatants after nanoparticles were prepared at the indicated mass ratios of protein to chitosan; (C) relative density of free proteins; (D) SDS-PAGE analysis of free proteins in supernatants after nanoparticles were prepared at the mass ratio of 1,024:1 (HTCC: protein) under different temperatures; (E) the relative density of free proteins. The protein supernatants were separated by SDS-PAGE and transferred to PVDF membranes, and the protein bands were visualized by ponceau staining. HTCC, N-(2-hydroxy) propyl-3-trimethyl ammonium; SDS-PAGE, sodium dodecyl sulfate polyacrylamide gel electrophoresis; PVDF, polyvinylidene fluoride.

from different encapsulation systems were run through sodium dodecyl sulfate-polyacrylamide gel electrophoresis. As shown in Figure $1 B, C$, when the mass ratio of protein to HTCC was 1:1, no clear protein encapsulation was found, because free dsNKG2D-IL-15's quantity was similar to that of the protein alone. If the ratio reached 1:512, the encapsulation rate increased to approximately $70 \%$. Therefore, when we use $1 \mu \mathrm{g}$ protein, the HTCC mass should be at least $512 \mu \mathrm{g}$. This condition is not feasible in protein encapsulation based on the HTCC itself. The effects of temperature on the encapsulation rate were also compared at 4,16 , or $25^{\circ} \mathrm{C}$. We did not observe any significant changes of encapsulation rate under different temperatures (Figure 1D,E).

\section{TPP facilitates the encapsulation of HTCC nanoparticles}

TPP can bind with the positively charged ions in chitosan, which significantly promotes chitosan encapsulating with RNA (14). During TPP addition to prepare the nanoparticles, the encapsulation rate sharply increased to more than $80 \%$, and the best encapsulation was obtained at the ratio (chitosan to protein) of 1:1 (Figure 2A,B). The size of protein nanoparticle was measured by both electric microscopy and dynamic light scattering. The protein nanoparticle displayed spherical shape with a diameter from 200 to $400 \mathrm{~nm}$ (Figure $2 C, D$ ). The zeta-potential value was $15.6 \pm 4.82 \mathrm{mV}$ (Figure $2 E)$. Thus, TPP could promote efficiency to generate the dsNKG2D-IL-15 protein nanoparticle based on HTCC.

\section{Protein releasing from nanoparticles}

dsNKG2D-IL-15 protein released from the HTCC-TPP nanoparticle was analyzed. Dialysis buffers were collected at indicated time courses. The results of electrophoresis showed that the protein release peaked after the protein nanoparticles were placed into the phosphate-buffered saline (PBS) for $72 \mathrm{~h}$ (Figure $3 A, B)$. The nanoparticles were mixed with the mononuclear cells of the spleen from normal mice to ensure that the dsNKG2D-IL-15 released from nanoparticles exhibited biological activity. As IL-15 promotes proliferation and survival of $\mathrm{CD}^{+} \mathrm{T}$ and NK cells, the number of changes of splenocytes treated by the protein nanoparticle reflected the function of the released protein. Expectedly, splenocytes treated with the protein nanoparticles at each dose for $72 \mathrm{~h}$ obtained higher cell numbers compared with vacant HTCC (Figure 3C). The apoptosis of splenocytes was also evaluated after the protein nanoparticle treatment. As shown in Figure $3 D, E$, low apoptosis was observed in splenocytes treated with $5 \mu \mathrm{g}$ nanoparticles (Figure 3D,E). Therefore, dsNKG2D- 
A

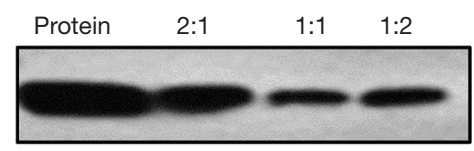

B

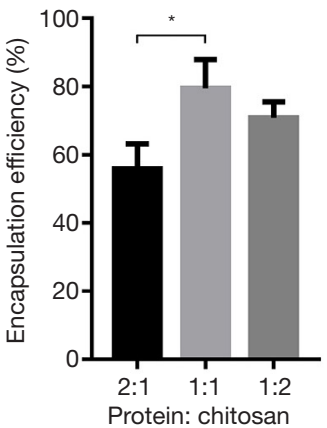

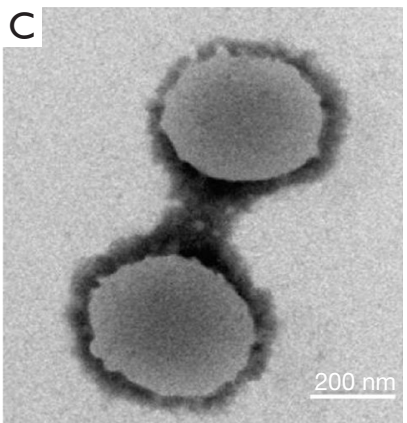
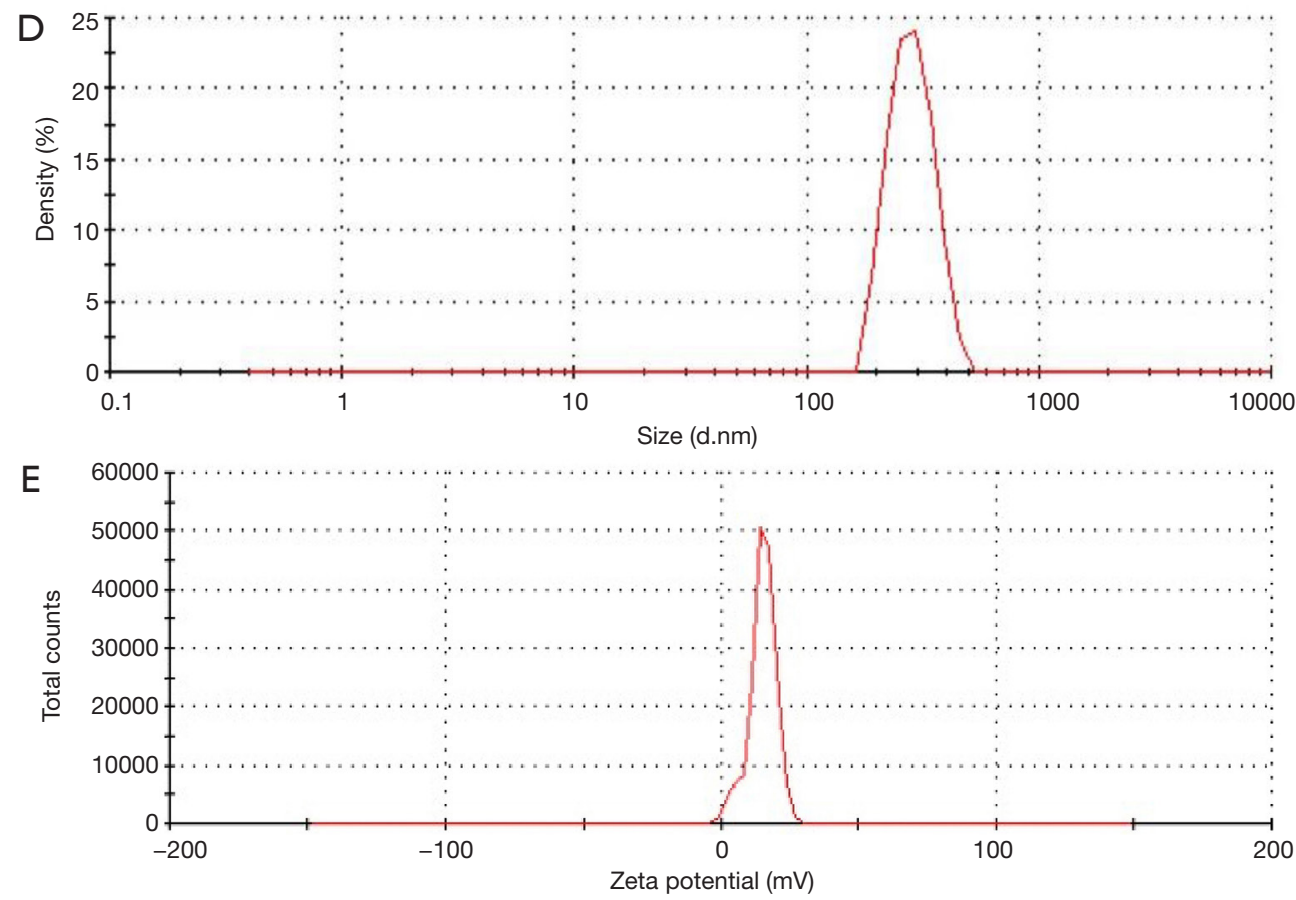

Figure 2 Characterization of dsNKG2D-IL-15 protein-nanoparticle based on HTCC and TPP. Adding TPP into the reactive system at the ratio of 10:1 (TPP:HTCC). (A) SDS-PAGE analysis of free proteins in supernatants after nanoparticles were generated; (B) the encapsulation rates of indicated mass ratios of HTCC to protein. $\mathrm{n}=3,{ }^{*}, \mathrm{P}<0.05$; (C) morphology and size detected by transmission electronic microscope; (D) size; (E) zeta-potential distribution. HTCC, N-(2-hydroxy) propyl-3-trimethyl ammonium; TPP, tripolyphosphate; SDS-PAGE, sodium dodecyl sulfate polyacrylamide gel electrophoresis.

IL15 could not only be easily released from the HTCC-TPP nanoparticle, but its activity on the lymphocytes could be retained.

\section{Cytotoxic effects of HTCC nanoparticles ex vivo}

The cytotoxicity of nanoparticles on RAW264.7 or B16MICA cells was detected by MTS/PMS method. No notable change in the chitosan nanoparticle was found between 1.25 and $2.5 \mu \mathrm{g}$ doses and culture medium. However, the $5.0 \mu \mathrm{g}$ dose exerted toxic effects on B16BL6-
MICA but not on RAW264.7 cells (Figure 4A,B). The linear relationship between the nanoparticle quantity and inhibition rate and also $\mathrm{IC}_{50}$ of nanoparticles of each cell type are shown in Figure 4C,D. Thus, low-dose HTCC nanoparticle is biologically safe, but high-dose HTCC nanoparticle has some cytotoxic effects.

\section{Stimulation of protein nanoparticles on $\mathrm{NK}$ and $C D 8^{+} \mathrm{T}$ cells ex vivo}

The stimulatory effects of dsNKG2D-IL-15 nanoparticle 


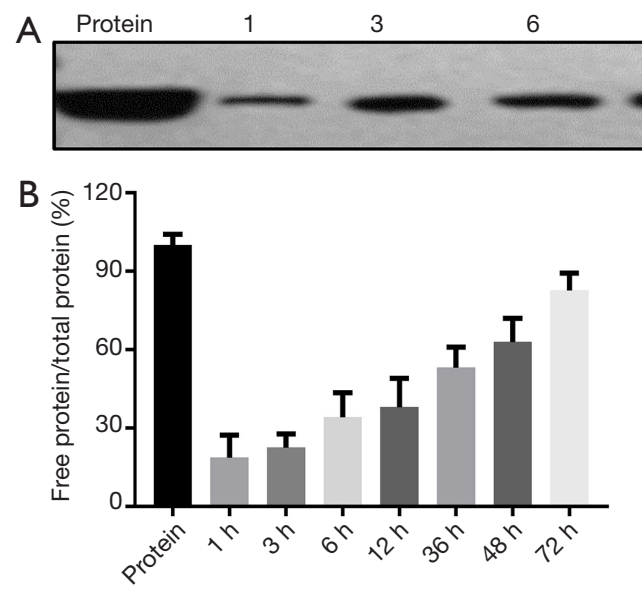

$\begin{array}{lll}12 & 24 & 48\end{array}$

$72(\mathrm{~h})$
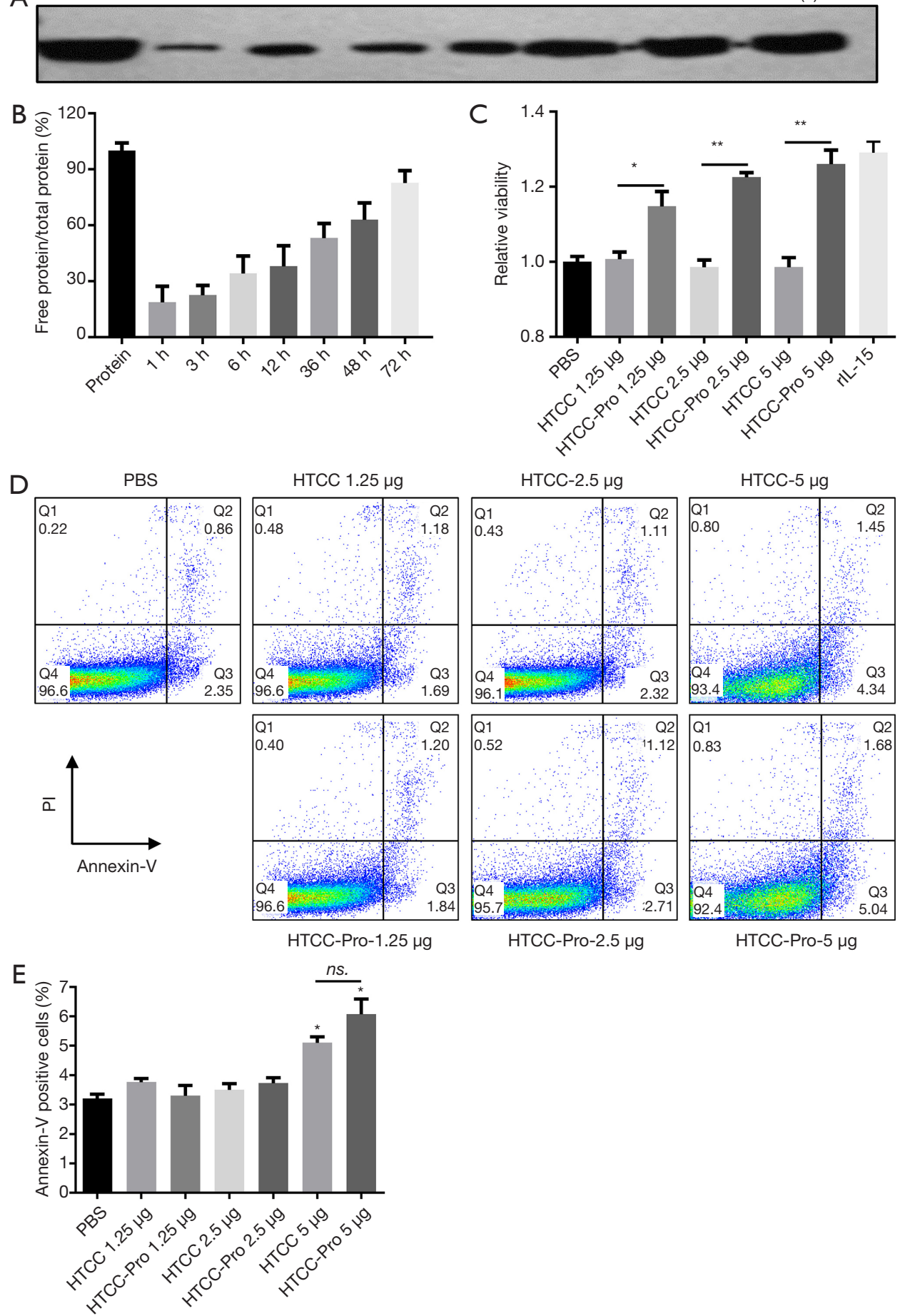

Figure 3 Release features of dsNKG2D-IL-15 protein-nanoparticle. (A) SDS-PAGE analysis of free proteins in dialysates after nanoparticles were added into PBS at the indicated time points; (B) relative density of free proteins, $\mathrm{n}=3$; (C) relative viability measured by MTS/PMS kit after splenocytes were mixed with protein-HTCC nanoparticle. $\mathrm{n}=6,{ }^{*}, \mathrm{P}<0.05$; ${ }^{* *}, \mathrm{P}<0.01$; (D) cells apoptosis measured by Annexin-V-PI stain kit; (E) statistical analysis of rate of Annexin- $\mathrm{V}$ positive cell. $\mathrm{n}=6$, Each experiment was performed thrice. ${ }^{*}, \mathrm{P}<0.05$; $\mathrm{ns}, \mathrm{no}$ significance. SDS-PAGE, sodium dodecyl sulfate polyacrylamide gel electrophoresis; PBS, phosphate buffer saline; MTS, 3-(4,5-dimethylthiazol-2-yl)-5(3-carboxymethoxyphenyl)-2-(4-sulfophenyl)-2H-tetrazolium, inner salt]; PMS, phenazine methosulfate; HTCC, N-(2-hydroxy) propyl-3trimethyl ammonium. 

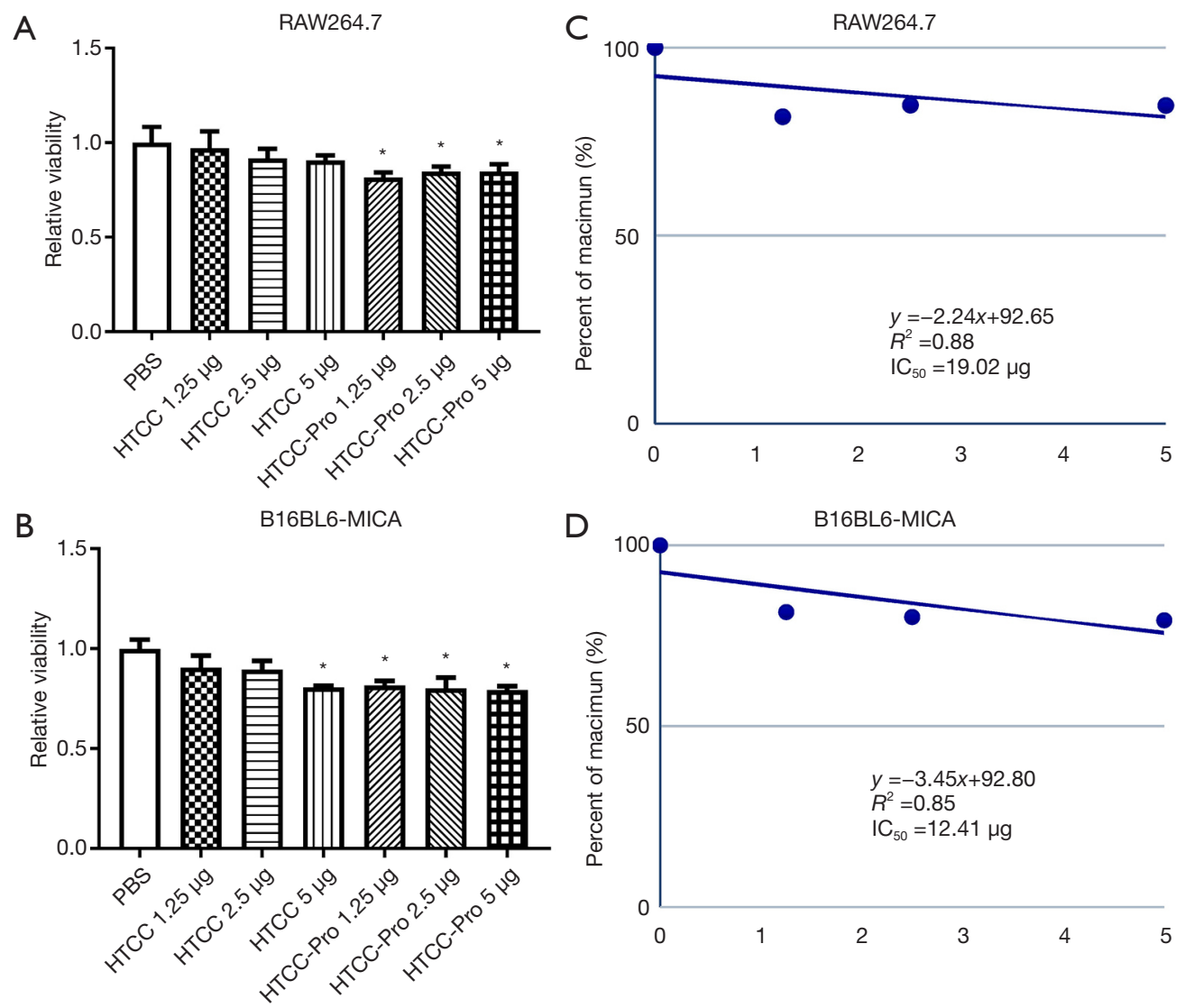

Figure 4 Cytotoxic effects of nanoparticle. (A) Variations in RAW264.7 or (B) B16BL6-MICA cell relative viability determined by MTS/ PMS kit after cell incubation with indicated quantities of protein-HTCC nanoparticles for 5 days. numbers of cells treated by nanoparticles were assayed by the absorbance at OD490 $\mathrm{nm}$. Linear relationship of nanoparticle quantity with inhibition rate of each cell type (C,D). $\mathrm{n}=6$, *, $\mathrm{P}<0.05$. MTS, 3-(4,5-dimethylthiazol-2-yl)-5-(3-carboxymethoxyphenyl)-2-(4-sulfophenyl)-2H-tetrazolium, inner salt]; PMS, phenazine methosulfate; HTCC, N-(2-hydroxy) propyl-3-trimethyl ammonium.

on $\mathrm{NK}$ and $\mathrm{CD}^{+} \mathrm{T}$ cells were then analyzed. When the protein nanoparticle was incubated with lymphocytes for $72 \mathrm{~h}$, NK cell showed enhanced CD69 expression (a surface marker of activation) and IFN- $\gamma$ production compared with cells treated with PBS or vacant HTCC. The cytotoxicity of NK cell was also significantly higher in the protein nanoparticle treatment than that of the PBS or vacant HTCC group. This result was evaluated by the degranulation activity based on CD107a staining (Figure $5 A$ ). Similar changes of $\mathrm{CD} 8^{+} \mathrm{T}$ cells were found after the treatments, as shown in Figure 5 B. Thus, the dsNKG2D-IL-15-nanoparticle treatment promoted the functions of $\mathrm{NK}$ and $\mathrm{CD} 8^{+} \mathrm{T}$ cells ex vivo.

\section{Antitumor effects mediated by protein nanoparticles in vivo}

We analyzed whether the treatment of dsNKG2D-IL-15 nanoparticle could suppress tumor growth in vivo. After B16BL6-MICA cells were subcutaneously transplanted into the back of mice for 5 days, PBS, vacant HTCC, dsNKG2D-IL-15-HTCC nanoparticle, or dsNKG2DIL-15 alone was peritoneally injected into the mice twice per week. We did not observe clear symptoms, including diarrhea, hair loss, or irritation, among all mice. DsNKG2D-IL-15-HTCC-TPP nanoparticle treatment obtained the highest tumor growth inhibition (Figure 6A). The tumor-bearing mice treated by the dsNKG2D-IL-15 nanoparticle had the longest period of survival (Figure 6B). Given that melanoma does not form solid tumors in the skin, we can only analyze the functional changes of the $\mathrm{NK}$ and $\mathrm{CD} 8^{+} \mathrm{T}$ cells of the spleens. Treatment with dsNKG2D-IL-15 nanoparticle increased the frequency of $\mathrm{NK} 1.1^{+} \mathrm{CD} 69^{+}$and NK1.1 $1^{+} \mathrm{NKG}_{2} \mathrm{D}^{+}$cells compared with PBS or vacant HTCC (Figure 6C). CD44 is a cell surface 

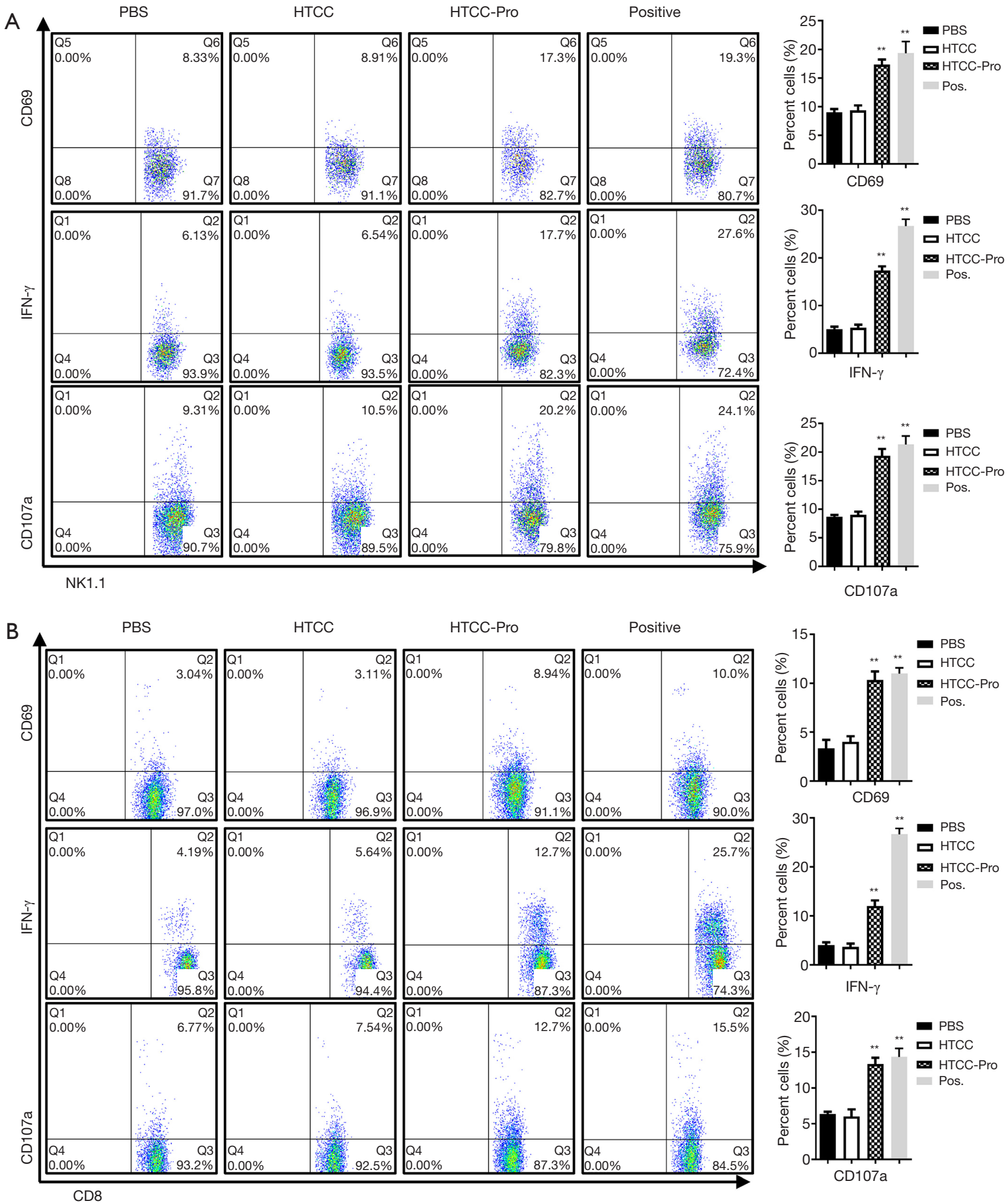

Figure 5 Stimulation of $\mathrm{NK}$ and CD8 ${ }^{+} \mathrm{T}$ cells by nanoparticle. The CD69 and CD107a expression levels of splenic (A) NK1.1 ${ }^{+}$or (B) CD8 ${ }^{+}$ $\mathrm{T}$ cells after co-cultured with nanoparticle $(2.5 \mu \mathrm{g})$ were detected by flow cytometry. IFN- $\gamma$ production was measured by flow-cytometry intracellular staining. Eecombinant IL-15 (25 ng/mL) used as positive control for CD69 and CD107a assay. Cell Stimulation Cocktail (eBioscience) as positive control used in IFN- $\gamma$ detection. Each experiment was performed thrice. $\mathrm{n}=6,{ }^{* *}, \mathrm{P}<0.01$. NK, natural killer. 

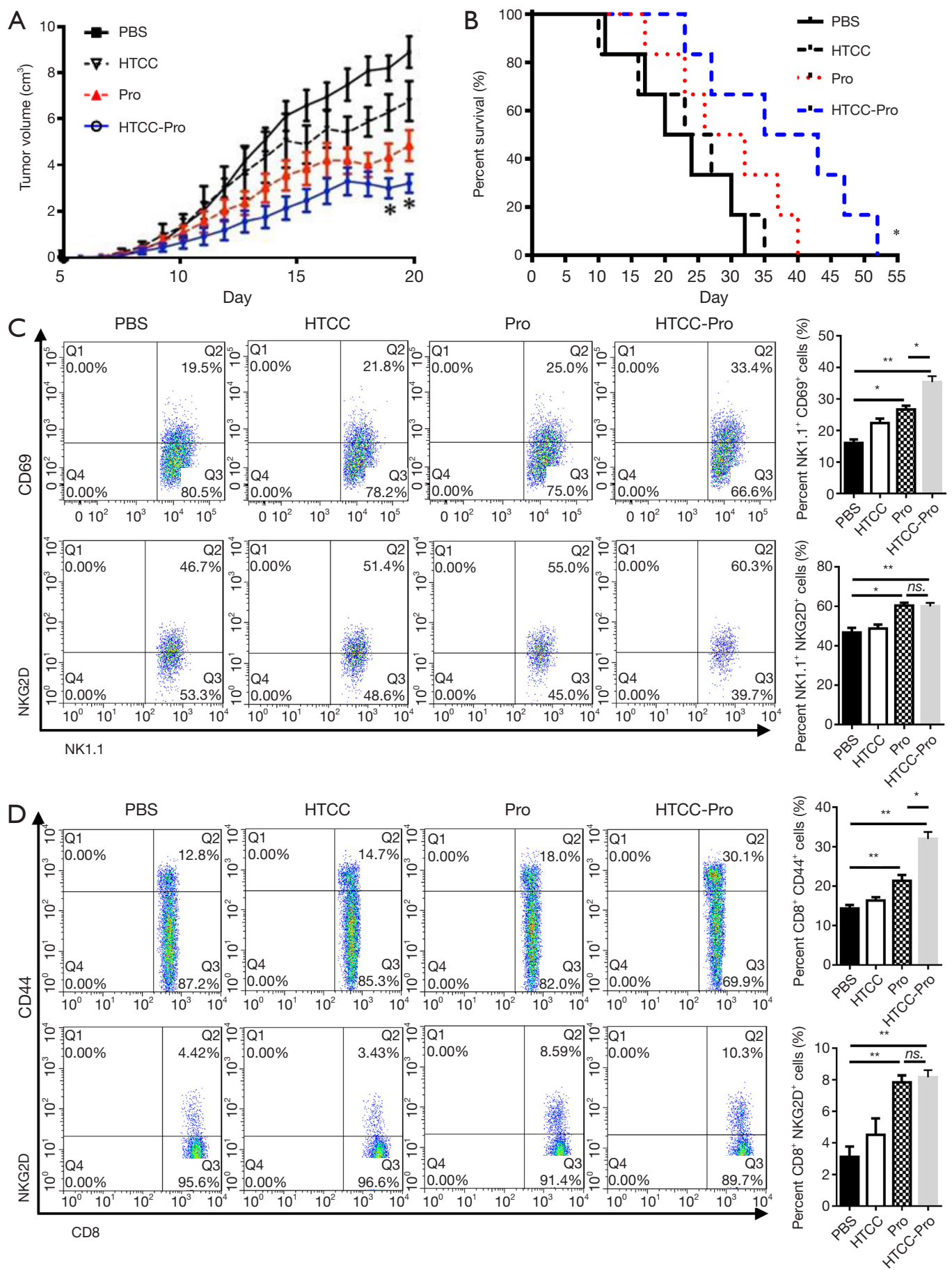

Figure 6 Anti-tumor activity mediated by dsNKG2D-IL-15 protein-HTCC nanoparticle. After the B16BL6-MICA cells were injected subcutaneously into the back of C57BL/6 mice for 5 days, protein-HTCC nanoparticles were peritoneally injected into mice every 3 days. Tumor growth was measured daily and expressed as the mean standard deviation (A); mouse deaths were documented over time. Data were presented in Kaplan-Meier survival curves (B). Some mice were sacrificed on day 20, and their spleens were collected. The frequencies of $\mathrm{NK} 1.1^{+} \mathrm{CD} 69^{+}$and $\mathrm{NK} 1.1^{+} \mathrm{NKG} 2 \mathrm{D}^{+}(\mathrm{C}), \mathrm{CD}^{+} \mathrm{CD} 44^{+}$, and $\mathrm{CD}^{+} \mathrm{NKG} 2 \mathrm{D}^{+}$cells (D) in spleens were detected by flow cytometry. The left panel shows the representative results of flow cytometry. The right panel shows the statistical data. The experiment was conducted twice. $\mathrm{n}=6,{ }^{*}, \mathrm{P}<0.05$, **, $\mathrm{P}<0.01$. HTCC, N-(2-hydroxy) propyl-3-trimethyl ammonium; NK, natural killer; ns, no significance. 
marker of activated and memory cells (15). NKG2D is only expressed by activated $\mathrm{CD} 8^{+} \mathrm{T}$ cells in mice (16). Similarly, higher frequencies of $\mathrm{CD} 8^{+} \mathrm{CD} 44^{+}$and $\mathrm{CD} 8^{+} \mathrm{NKG} 2 \mathrm{D}^{+}$ cells treated by dsNKG2D-IL-15-HTCC nanoparticle were found in tumor-bearing mice compared with PBS or vacant HTCC (Figure 6D). However, no significant changes of $\mathrm{NK} 1.1^{+} \mathrm{NKG} 2 \mathrm{D}^{+}$and $\mathrm{CD} 8{ }^{+} \mathrm{NKG}_{2} \mathrm{D}^{+}$cell frequencies were found between the treatments of dsNKG2D-IL15-HTCC nanoparticle and dsNKG2D-IL-15 protein alone. On the contrary, the frequencies of NK1.1 $1^{+} \mathrm{CD} 69^{+}$ and $\mathrm{CD} 8^{+} \mathrm{CD} 44^{+}$cells were higher in the treatment of dsNKG2D-IL-15-HTCC nanoparticle than that of dsNKG2D-IL-15 alone. Thus, dsNKG2D-IL-15-HTCC nanoparticle could exert significant antitumor effects by activating the function of $\mathrm{NK}$ and $\mathrm{CD} 8^{+} \mathrm{T}$ cells.

\section{Discussion}

In this study, the NKG2D-IL-15 fusion protein, which can bind with the NKG2D ligands (e.g., MICA) of tumor cells and activate lymphocytes simultaneously, was encapsulated into HTCC-TPP-based nanoparticles. Although chitosan was modified to have quaternary ammonium in the backbone, the encapsulation efficiency was very low without the TPP addition. The protein nanoparticle was positively charged with the size of approximately 200-400 nm. NKG2D-IL-15 could be efficiently released from the nanoparticle within $72 \mathrm{~h}$, and the released protein maintained its biological activity for stimulating lymphocytes. Protein nanoparticle exerted better effects on the inhibition of tumor growth and prolonged the survival of tumor-bearing mice in vivo than the NKG2D-IL-15 fusion protein alone. This nanoparticle could be potentially used as a new reagent for tumor therapy.

Several factors are involved in nanoparticle construction, including concentration and molecular weight of chitosan, encapsulant concentration, polymer $\mathrm{pH}$, anionic crosslinker solutions, ratio chitosan/anionic crosslinker, speed, and duration of agitation or mixing $(2,17,18)$. TPP addition before ionic gelation facilitated the encapsulation of both hydrophilic and hydrophobic drugs with excellent drugloading capacity and encapsulation efficiency (14). We confirmed that the encapsulation efficiency of HTCCTPP nanoparticles was significantly higher $(85-95 \%)$ than that of native chitosan nanoparticles (10-20\%). In addition, the dsNKG2D-IL-15-HTCC-TPP nanoparticle obtained good protein release in vitro. The nanoparticle's stability is generally affected by $\mathrm{pH}$ value, temperature, and storage duration (19,20).

DsNKG2D-IL-15 fusion protein showed more efficiency in suppressing the growth of colon and gastric cancers than soluble IL-15 $(3,4)$. Here, dsNKG2D-IL-15 protein nanoparticle displayed stronger antitumor effects in vivo compared with dsNKG2D-IL-15 protein alone. We did not observe significant changes of splenic NK1.1 ${ }^{+} \mathrm{NKG}_{2} \mathrm{D}^{+}$ and $\mathrm{CD} 8^{+} \mathrm{NKG}_{2} \mathrm{D}^{+} \mathrm{T}$ cells between the treatments of dsNKG2D-IL-15 nanoparticle and protein. This finding inferred that dsNKG2D-IL-15-HTCC nanoparticle mediated the antitumor effects dependent on similar molecular mechanisms with dsNKG2D-IL-15 protein in vivo. However, dsNKG2D-IL-15-HTCC nanoparticle was potentially distributed in the target tumor tissues and played a lasting effect (e.g., promoting the generation of memory $\mathrm{CD} 8^{+} \mathrm{T}$ cells) by slow release in vivo $(3,6)$.

A dsNKG2D-IL-15 gene nanoparticle was developed to antagonize the growth of colon cancer in mice. dsNKG2DIL-15 protein nanoparticle has some advantages compared with the gene nanoparticle. A prerequisite for transporting genes based on nanomaterials is that the nanoparticles are phagocytosed by the cells in the body. In general, the phagocytosis efficiency in vivo is hard to predict (21). The genes in the intracellular nanoparticles must be released into the cytoplasm and efficiently expressed in the cells. If the genes encode soluble proteins, then these proteins are expected to be released to the context of cells (22). Finally, when the protein nanoparticle enters the body, it preferentially migrates to and is kept in tumor tissues due to the EPR effect (10). Even if the nanoparticle releases some quantities of dsNKG2D-IL-15 in the peripheral blood, this protein tends to be distributed in tumor tissues, as shown in our study (3).

If the clinical use of this new protein nanoparticle is considered, several questions need to be considered. To avoid in vivo toxicities mediated by chitosan, relatively low dose should be used in tumor-bearing mice. We wondered whether we can obtain better antitumor effects at high dose. Meanwhile, the stringent toxic effects of the nanoparticle in vivo should be further clarified $(23,24)$. Whether the dsNKG2D-IL-15 nanoparticle plays an important role against other tumor types also requires further analysis.

\section{Conclusions}

DsNKG2D-IL-15 fusion protein could be encapsulated into HTCC-TPP nanoparticles. The protein can be efficiently released from the nanoparticle while retaining 
its biological activity. The dsNKG2D-IL-15 protein nanoparticle exhibited notable effects on lymphocyte activation and tumor inhibition, thereby indicating that the protein nanoparticle can be developed for tumor therapy in clinical practice.

\section{Acknowledgments}

Funding: This work was supported by the National Natural Science Foundation of China (Nos. 81671547, 81471547, $81873867,81873866)$, the "Six peaks" Talent Project, and the "333" Talent Project in Jiangsu Province, the Natural Science Foundation of Jiangsu Province (BK20180925, BK20160479).

\section{Footnote}

Conflicts of Interest: The authors have completed the ICMJE uniform disclosure form (available at http://dx.doi. org/10.21037/tcr.2019.09.36). The authors have no conflicts of interest to declare.

Ethical Statement: The authors are accountable for all aspects of the work in ensuring that questions related to the accuracy or integrity of any part of the work are appropriately investigated and resolved. All applicable international and national guidelines for the care and ethics of animals were followed. All animal experimental protocols were approved by the Animal Care and Ethics Committee of Yangzhou University, Yangzhou, China AApproval ID: SYXK [Su] 2017-0044\}.

Open Access Statement: This is an Open Access article distributed in accordance with the Creative Commons Attribution-NonCommercial-NoDerivs 4.0 International License (CC BY-NC-ND 4.0), which permits the noncommercial replication and distribution of the article with the strict proviso that no changes or edits are made and the original work is properly cited (including links to both the formal publication through the relevant DOI and the license). See: https://creativecommons.org/licenses/by-nc-nd/4.0/.

\section{References}

1. Kamath PR, Sunil D. Nano-Chitosan Particles in Anticancer Drug Delivery: An Up-to-Date Review. Mini Rev Med Chem 2017;17:1457-87.

2. Zhang X, Yang X, Ji J, et al. Tumor targeting strategies for chitosan-based nanoparticles. Colloids Surf B Biointerfaces 2016;148:460-73.

3. Chen Y, Chen B, Yang T, et al. Human fused NKG2DIL-15 protein controls xenografted human gastric cancer through the recruitment and activation of NK cells. Cell Mol Immunol 2017;14:293-307.

4. Xia Y, Chen B, Shao X, et al. Treatment with a fusion protein of the extracellular domains of NKG2D to IL15 retards colon cancer growth in mice. J Immunother 2014;37:257-66.

5. Carapito R, Bahram S. Genetics, genomics, and evolutionary biology of NKG2D ligands. Immunol Rev 2015;267:88-116.

6. Jelenčić V, Lenartić M, Wensveen FM, et al. NKG2D: A versatile player in the immune system. Immunol Lett 2017;189:48-53.

7. Muntasell A, Ochoa MC, Cordeiro L, et al. Targeting NK-cell checkpoints for cancer immunotherapy. Curr Opin Immunol 2017;45:73-81.

8. Robinson TO, Schluns KS. The potential and promise of IL-15 in immuno-oncogenic therapies. Immunol Lett 2017;190:159-68.

9. Yan C, Jie L, Yongqi W, et al. Delivery of human NKG2D-IL-15 fusion gene by chitosan nanoparticles to enhance antitumor immunity. Biochem Biophys Res Commun 2015;463:336-43.

10. Tan L, Han S, Ding S, et al. Chitosan nanoparticlebased delivery of fused NKG2D-IL-21 gene suppresses colon cancer growth in mice. Int $\mathrm{J}$ Nanomedicine 2017;12:3095-107.

11. Faizuloev E, Marova A, Nikonova A, et al. Water-soluble $\mathrm{N}$-[(2-hydroxy-3-trimethylammonium)propyl]chitosan chloride as a nucleic acids vector for cell transfection. Carbohydr Polym 2012;89:1088-94.

12. Zhang $X$, Geng $X$, Jiang $H$, et al. Synthesis and characteristics of chitin and chitosan with the (2-hydroxy3 -trimethylammonium)propyl functionality, and evaluation of their antioxidant activity in vitro. Carbohydr Polym 2012;89:486-91.

13. Desai KG. Chitosan Nanoparticles Prepared by Ionotropic Gelation: An Overview of Recent Advances. Crit Rev Ther Drug Carrier Syst 2016;33:107-58.

14. Xiao B, Ma P, Ma L, et al. Effects of tripolyphosphate on cellular uptake and RNA interference efficiency of chitosan-based nanoparticles in Raw 264.7 macrophages. J Colloid Interface Sci 2017;490:520-8.

15. Ali AJ, Abuelela AF, Merzaban JS. An Analysis of Trafficking Receptors Shows that CD44 and P-Selectin 
Glycoprotein Ligand-1 Collectively Control the Migration of Activated Human T-Cells. Front Immunol 2017;8:492.

16. McQueen B, Trace K, Whitman E, et al. Natural killer group 2D and CD28 receptors differentially activate mammalian/mechanistic target of rapamycin to alter murine effector CD8+ T-cell differentiation. Immunology 2016;147:305-20.

17. Afsharzadeh M, Hashemi M, Mokhtarzadeh A, et al. Recent advances in co-delivery systems based on polymeric nanoparticle for cancer treatment. Artif Cells Nanomed Biotechnol 2018;46:1095-110.

18. Ahmed TA, Aljaeid BM. Preparation, characterization, and potential application of chitosan, chitosan derivatives, and chitosan metal nanoparticles in pharmaceutical drug delivery. Drug Des Devel Ther 2016;10:483-507.

19. Jonassen H, Kjoniksen AL, Hiorth M. Stability of chitosan nanoparticles cross-linked with tripolyphosphate. Biomacromolecules 2012;13:3747-56.

20. Morris GA, Castile J, Smith A, et al. The effect of

Cite this article as: Chen R, Ding Y, Xi J, Lu G, Xiao W, Ding Y, Qian L, Lin Z, Gong W. NKG2D-IL-15 fusion protein encapsulated in N-[(2-hydroxy-3-trimethylammonium) propyl] chitosan chloride retards melanoma growth in mice. Transl Cancer Res 2019;8(6):2230-2241. doi: 10.21037/tcr.2019.09.36 prolonged storage at different temperatures on the particle size distribution of tripolyphosphate (TPP) - chitosan nanoparticles. Carbohydrate Polymers 2011;84:1430-4.

21. Zheng H, Tang C, Yin C. The effect of crosslinking agents on the transfection efficiency, cellular and intracellular processing of DNA/polymer nanocomplexes. Biomaterials 2013;34:3479-88.

22. Du H, Yang X, Zhai G. Design of chitosan-based nanoformulations for efficient intracellular release of active compounds. Nanomedicine (Lond) 2014;9:723-40.

23. Das J, Choi YJ, Song H, et al. Potential toxicity of engineered nanoparticles in mammalian germ cells and developing embryos: treatment strategies and anticipated applications of nanoparticles in gene delivery. Hum Reprod Update 2016;22:588-619.

24. Parveen R, Shamsi TN, Fatima S. Nanoparticles-protein interaction: Role in protein aggregation and clinical implications. Int J Biol Macromol 2017;94:386-95. 